\title{
Desarrollo social y educación superior
}

\section{Social Development and Higher Education}

\author{
Carolina España Chavarría ${ }^{l}$ \\ Escuela de Secretariado Profesional \\ Universidad Nacional \\ Heredia, Costa Rica \\ cespanac@yahoo.com.mx
}

Recibido 12 de abril de 2011 • Aceptado 26 de agosto de 2011

\begin{abstract}
Resumen. El presente ensayo tiene por objetivo reflexionar sobre el quehacer de la universidad pública costarricense y su responsabilidad en la formación para el desarrollo social. Lo anterior, entendido como uno de los múltiples retos que enfrenta la educación superior, de cara a las demandas que la función del conocimiento posee en el presente, y a la relación de estas con el desarrollo autónomo del conocimiento. Su planteamiento, defiende algunos asuntos débilmente abordados en estudios previos, y que se constituyen en elementos esenciales para una formación significativa, funcional y de impacto social, como son: a) La ética en la organización, b) La autoeducación de la universidad, c) La incidencia de las políticas curriculares en las prácticas promovidas, d) La transformación de la cultura docente para contribuir a mejorar la práctica, y e) La construcción del conocimiento para fundamentar criterios, tomar decisiones, solucionar problemas y construir proyectos de vida.
\end{abstract}

Palabras claves. Sociedad, aprendizaje significativo, educación, práctica docente

Abstract. This essay has as its main objective to reflect on the duty of the Costa Rican public university and its responsibility to educate in order to foster social development, which is understood as one of the multiple challenges that the higher education faces due to the demands imposed on the operation of knowledge in the present and the relation of such demands with independent knowledge development. In addition, a defense is made of some issues that have been approached weakly in previous studies, issues that become part of the essential elements for promoting a meaningful and functional education that has social impact, elements such as the following: a) Ethics in the organization, b) The university's selfeducation, c) The effect of curricular policies on the practices being promoted, d) The transformation of the teaching culture to improve practice, and e) The construction of knowledge on which to base criteria, decision making, problem solving and the development of life projects.

Keywords. Society, Meaningful Learning, Education, Teaching Practice

\footnotetext{
Egresada del Doctorado en Políticas y Prácticas de Innovación Educativa para la Sociedad del Conocimiento por la Universidad de Almería, España. Magister en Políticas y Prácticas de Innovación Educativa para la Sociedad del Conocimiento por la Universidad de Almería, España. Magister en "Segundas Lenguas y Culturas con Énfasis en Inglés como Lengua extranjera para: Alumnado Adulto" por la Universidad Nacional, Costa Rica. Docente de Postgrado en el área de la educación y enseñanza del inglés. Actualmente labora en la Escuela de Secretariado Profesional de la Universidad Nacional. Cuenta con ponencias y asesorías internacionales realizadas en la USAC (Universidad de San Carlos, Guatemala):“Técnicas para la comunicación oral en inglés orientada a la locución", "Estrategias y metodologías de la enseñanza del idioma inglés", "Técnicas para la comunicación oral en inglés orientadas al periodismo". "Profesionalización del colectivo docente de la escuela de Lingüística".
} 


\section{Introducción}

La responsabilidad de la formación promovida por la universidad pública para contribuir al desarrollo social podría constituirse en uno más de los retos por asumir de cara a las demandas que el funcionamiento del conocimiento posee en el siglo XXI y a la relación de estas con el papel de la ciudadanía en el trato de las cuestiones suscitadas en el entorno.

De allí que sea posible coincidir en que:

(...) las grandes respuestas sobre el sentido de la educación y que se concretan en el tipo de ciudadano o ciudadana que queremos formar, son las razones de fondo que planean este debate y que configuran una determinada jerarquización de los saberes y su forma de presentarlos y organizarlos (...). (Zabala, 1999, p. 39)

A partir de lo anterior, la posibilidad de entender el objetivo de la educación estatal costarricense, el cual se centra en la construcción del conocimiento para fundamentar criterios, tomar decisiones, solucionar problemas y construir proyectos de vida.

No obstante, para poder garantizar un proceso de formación autónomo y versado a construir aprendizajes sustanciales, Oliva y Henson (1989) puntualizan en la necesidad de hacer a la universidad responsable de transformar, de manera urgente, su ética organizativa. Para estos autores, la dimensión ética desde donde se fundamente la reestructuración de la organización universitaria es parte de ese proceso de autoeducación requerido por la universidad y clave esencial en una formación promotora de capacidades amigables con los problemas del entorno. Es en el contexto universitario en donde se desarrollan seres comprometidos con el quehacer comunal, defensores de la inclusión social de los más vulnerables, favorecedores de la accesibilidad del conocimiento, respetuosos de la condición humana por sobre la material, agentes de transformación y personas creativas en la articulación de su profesión con las demandas y necesidades de su entorno.

Igualmente, se destaca la necesidad de emprender una lectura minuciosa de las políticas curriculares responsables de liderar la función universitaria, para poder así conocer y entender su incidencia en la práctica promovida y en la cultura docente.

\section{La transformación educativa y su incidencia en el modelo educativo promovido}

La construcción de un modelo educativo que se aproxime a las realidades del entorno se vislumbra como una necesidad que debe atender significativamente el proceso de socialización escolar y la promoción social. Además, de acuerdo con Tedesco (1999), se cree que dicho modelo es el responsable de promover el desarrollo personal y la construcción del conocimiento autónomo y reflexivo en el colectivo estudiantil, todo esto apegándose a principios éticos fortalecidos por bases teóricas que permitan fundamentar, conocer y entender las realidades surgidas de la vinculación Estado-Sociedad.

Coincidiendo con Gimeno y Pérez (1992), el modelo educativo que guía la práctica pedagógica se deriva de un modelo del saber democrático, el cual reflejaría los principios éticos, de equidad y 
sostenibilidad que garanticen la construcción de conocimientos basados en aprendizajes autónomos, con impacto social, propositivos y resolutivos, el asunto deriva en: ¿cómo lograrlo?

Lo anterior le exige a la universidad pública costarricense que tome conciencia de su responsabilidad como motor del desarrollo y le impone un replanteamiento de su misión y visión para seguir legitimándola, de allí la supuesta necesidad, a la cual apunta Torres (2006), de que los modelos sociales se analicen, se reflexionen sistemáticamente y se practiquen, para sacar a la luz las realidades y transformarlas en aprendizajes autónomos, significativos y funcionales, de los cuales se podrá aprender a aprender.

La tarea, según lo visto, es compleja; pero responde indudablemente al interés de la sociedad porque la educación contribuya a formar sujetos libres de pensamiento y de accionar, pero, ¿con qué intención? y, ¿cómo será posible llevarlo a cabo?

Se podría afirmar que su intencionalidad radica en brindarle al individuo una mejora sustancial de vida, personal y profesional, para lo cual será preciso la implementación de una filosofía de trabajo como herramienta de organización, capacitación, orientación y promoción de saberes interdisciplinarios, que se facilitan y construyen a través de un enfoque pedagógico basado en el desarrollo de competencias, permitiéndole a la persona fundamentar su criterio y así tomar decisiones que faciliten el prever y solucionar problemas, así como también construir y liderar proyectos a favor de su desarrollo personal y profesional.

Es decir, la sociedad costarricense le demanda a la universidad del estado una postura más allá de simple nicho de cultivo para el conocimiento y clama porque se convierta en una institución aprendiente, investigadora y transformadora de su accionar, ya que “(...) para que una escuela que enseña se convierta en una institución que aprende necesita estar articulada sobre unas características que hagan viable la cultura de la reflexión (...)” (Santos, 2001, p. 67).

Para que los centros públicos universitarios atiendan lo evidenciado por Santos, podría ser esencial no solo asumir el reto de vincular su hacer con los asuntos que aquejan a la sociedad costarricense, sino también convertirse en ejemplo de comunidad reflexiva, esto es, podría ser preciso que los centros de enseñanza superior implementen una estrategia o plan de acción educativo de impacto en los sectores de la sociedad (económico, político y social), mediante el impulso de la reflexión-acción en donde se conduzca a vivir en sociedad para pregonar su condición social. En este sentido, concebir a los centros de formación superior como entes llamados a velar por la puesta en marcha de transformaciones significativas y funcionales de su organización, estructura y procesos, como una de las condiciones para construir conocimiento en interacción con la sociedad.

La importancia otorgada a la indagación y al análisis en la enseñanza superior podría contribuir a romper con prácticas pedagógicas reproductoras, transmisoras y acríticas, permitiendo introducir un instrumento promotor de la construcción de conocimiento. Así se incidirá en el trato que se le dé a los valores, a la equidad, al asunto de género, a la accesibilidad, a la sostenibilidad, entre otros elementos que impactan la sociedad, puesto que "(...) toda enseñanza (...) debería ser estratégica, (...) concebida en una perspectiva a largo plazo, en el que cada acción se decide en función de su contribución esperada en la progresión óptima de los aprendizajes de cada uno" (Perrenoud, 2004, p. 33).

Por lo cual lo relevante de entender que la formación deberá ser guiada por un enfoque de enseñanza que promueva el desarrollo de capacidades básicas o competencias claves en el estudiantado, base que fundamentará el conocimiento desarrollado a la luz de la significancia y funcionalidad requerida para afrontar los retos que impone la vida. 
Igualmente, se valora como trascendental el hablar de la necesidad de un modelo de enseñanza como el enfoque por competencias, atendiendo al discurso que sobre este se maneja; pues es allí cuando la práctica pedagógica se ve impactada.

Por ende, no basta con que el Estado costarricense promueva cambios educativos para que estos surtan efecto. Dichas transformaciones deberán ir de la mano del convencimiento de quienes las han de promover a partir de su puesta en marcha y valoración a la luz de otros enfoques y prácticas pedagógicas tradicionalmente utilizadas, ya que así podría surgir el interés por innovar.

\section{Cultura educativa: transformación e impacto en la promoción de aprendizajes}

Partiendo de la existencia de un estrecho ligamen entre el desarrollo del conocimiento y la cultura educativa, esta última podría resultar de ideas convertidas en acciones fosilizadas sin un debido y continuo análisis.

El tipo de socialización desarrollada en los centros universitarios no surge de la nada, es el resultado de formas de ser y hacer, las cuales han sido moldeadas por años y socialmente concebidas como ordenanzas que condicionan la formación y, consecuentemente, el ser del individuo.

No obstante, así como la cultura educativa es construida como parte del accionar en sociedad, igualmente podrá ser transformada y reconducida, puesto que enriquece e influye en el accionar del sujeto.

La cultura educativa no supone limitar al individuo a un solo ángulo de percepción, por el contrario, autores como Freire (2002) apuntan a su finalidad para el desarrollo de capacidades, en el estudiantado, orientadas a la indagación para entender y desenredar las problemáticas del entorno, pero desde el debido fundamento que el razonamiento, la construcción del entendimiento y la globalidad que el conocimiento facilita. Ahora bien, si todo parece estar claro, ¿por qué todavía el asunto de la transformación de la cultura docente es un tema de interés cuando se atiende a la formación universitaria?

En este sentido, para que la cultura educativa, y en especial aquella que caracteriza al colectivo docente, sea transformada, se considera fundamental poner la mira en el cambio de actitud de dicho profesional, quien valorará su función formativa desde la integración interdisciplinar de saberes, lo cual fundamentará la relación entre teoría y práctica de manera significativa y congruente con las realidades de la sociedad.

Igualmente, se cree crucial que el profesorado ponga atención a los contextos de aprendizaje para que se constituyan en espacios libres de prejuicios y ejemplificaciones utópicas, caracterizados más bien por su función facilitadora en la construcción del conocimiento, el análisis y el contraste de las realidades, cada una de estos objetivos fundamentales del proceso formativo y producto de un accionar educativo, en donde la investigación ya no sería vista como un área del trabajo universitario aislada, sino como complemento e insumo del proceso de enseñanza-aprendizaje del estudiantado.

Tanto la integración de saberes, el ambiente de aprendizaje nutrido por la realidad y la investigación como guía del proceso, contribuyen sustancialmente en la toma de decisiones del sujeto en formación. El proceso para la construcción del conocimiento adoptaría una naturaleza indagatoria, de resultados mejorables, producto de su inevitable evolución y le enseñaría al estudiantado que cuando aprende, se está en la capacidad de reconstruir, validar, desechar o 
transformar la teoría, así como también las realidades del entorno para que de estas se genere conocimiento funcional.

Lo anterior pone en clara evidencia la urgente necesidad de un colectivo docente propositivo, deliberante, no pasivo y amigable con los modelos pedagógicos contemporáneos, aquellos versados en otorgarle a la construcción del conocimiento su funcionalidad y autonomía para evitar que los contextos de aprendizaje universitarios sean espacios de "enseñanza" fuera de contexto, despersonalizada y sin utilidad para enfrentar los desafíos de la vida.

\section{La promoción de aprendizajes relevantes: interdisciplinaridad, investigación y práctica pedagógica}

La investigación como guía del proceso formativo facilita el análisis de la práctica, permitiéndole al docente fundamentar el ejercicio de su labor desde una dimensión que va más allá de la producción de “(...) buenos resultados académicos, por la evitación de los conflictos, y por el mantenimiento del orden y de las costumbres" (Santos, 2001, p. 38 ). El indagar permite reflexionar y los artífices del proceso educativo deberán adoptar estas como prácticas para el entendimiento de la vida.

La necesidad de conocer del docente y del alumnado no podrá sustraerse de una promoción de aprendizajes globales, que conducen a interrelacionar asuntos de formas diversas, ampliando simultáneamente el corpus del conocimiento y permitiendo sedimentar criterios producto de la construcción de teoría a partir de la práctica.

La interdisciplinaridad, como los hilos que hilvanan la construcción del conocimiento y la formación docente, como elemento fundamental para una práctica significativa, se asocian a las múltiples demandas de la universidad contemporánea y, en este caso en particular, la costarricense, en el marco de la transversalidad como elemento clave de la formación del estudiantado. Esta deberá ser transformada por el colectivo docente en conocimiento enseñable, entendible y funcional para su crecimiento como profesional y como parte esencial de la formación del estudiantado.

Se coincide entonces en que "(...) La interdisciplinaridad es fundamentalmente un proceso y una filosofía del trabajo que se pone en acción a la hora de enfrentarse a los problemas y cuestiones que preocupan en cada sociedad (...)" (Torres, 2006, p. 67).

De allí la importancia de asumir la construcción del conocimiento como un proceso enriquecido y fortalecido por múltiples visiones emergidas del entorno, en donde la investigación protagoniza un papel determinante en la práctica formativa, promoviendo la indagación para su entendimiento a la luz del análisis del porqué, el para qué y el cómo acontecen las realidades evidenciadas.

A pesar de los múltiples aportes de la investigación a la práctica pedagógica, como herramienta que conduce el acto pedagógico, su protagonismo no puede ser garantizado, a no ser que forme parte de las ideas que conforman el discurso del colectivo docente sobre su función formadora. Al respecto, se concuerda con la afirmación de que “(...) cualquier propuesta encaminada a conseguir que alguien aprenda está condicionado o determinado por una idea, consciente o inconsciente, de persona y de sociedad" (Zabala, 1999, p. 36).

Partiendo de esta premisa, se cree determinante que los discursos se sometan a reflexión crítica, la misma, a la luz de los asuntos que embullen a la sociedad como parte fundamental para 
garantizar la calidad en la función educativa, la cual tiene como auténtica razón de ser el "(...) preparar a los ciudadanos y ciudadanas para comprender, juzgar e intervenir en su comunidad, de una manera responsable, justa, solidaria y democrática (...)” (Torres, 2006, p. 20).

\section{Retos de la política educativa y de la estructura del funcionamiento universitario}

La educación superior pública enfrenta el gran desafío de formar un colectivo capaz de interactuar proactivamente en la sociedad a la cual se debe, por lo tanto, de acuerdo con lo que establece Stenhouse (2004), es necesario contar con un currículo promotor de la crítica, el análisis, la inventiva y demás características fundamentales en apoyo a su razón de ser como orientación pedagógica.

El análisis de la política curricular a la cual se adscribe la educación superior pública en Costa Rica es uno más de los retos que ha de asumir, junto con la estructura de su funcionamiento universitario.

Se cree importante analizar la estructura del funcionamiento universitario y someter a consideración su reforma, esto por cuanto dicha institución ha de velar por el debido accionar de su gestión interna, la cual estaría impactando la acción pedagógica y la formación permanente del profesorado y alumnado, producto de la dicotomía que subyace del currículo oculto y el explícito, según se indica:

El curriculum explícito u oficial aparece claramente reflejado en las intenciones que, de una manera directa, indican las normas legales, los contenidos mínimos obligatorios o los programas oficiales, como los proyectos educativos de centro, [a su vez] el curriculum oculto hace referencia a todos aquellos conocimientos, destrezas, actitudes y valores que se adquieren mediante la participación en procesos de enseñanza y aprendizaje y, en general, en todas las interacciones que suceden día a día en las aulas y centros de enseñanza (...). (Torres, 2005, p. 198)

Tanto el currículo oculto como el explícito requieren de análisis y reflexión. Aunque su condición como instrumentos orientadores del discurso y de las acciones educativas manejadas por los artífices del proceso los hacen responsables de enlazar el acto formativo con las realidades del entorno, en lo concreto podría no ser tan factible dicha vinculación, por cuanto algunos profesionales parecieran estar llevando el currículum ofertado a su mera ejecución, obviando su significancia y funcionalidad.

Lo anterior conduce a preguntarse: ¿estará el problema de fondo asociado más a un asunto de formación por parte del docente? o, ¿será que es débil su posicionamiento ante la autonomía profesional que supone dirigir su función? La contestación a estas preguntas no permite un abordaje ligero, poco fundamentado o desligado del contexto nacional costarricense. Lo que sí cabe destacar es que la formación actualizada, continua y pertinente conduce al empoderamiento del conocimiento $\mathrm{y}$, consecuentemente, a un desarrollo de liderazgo académico esencial para hacer las adaptaciones pedagógicas y disciplinares asertivas - que toda oferta curricular requiere- de manera integrada a los asuntos que interesan y transforman la sociedad. 
Por tanto, el análisis de la estructura universitaria permitirá evidenciar las rutas que facilitarán el acercamiento de esta a su comunidad, constituyéndose en elemento significativo para que logre formar en ella y desde ella, es decir, transformándose en modelo de micro-sociedad en el cual el profesor y la universidad, como entidad educadora, logren promover de la mano del currículo ofertado “(...) de la cual se construye y se gestiona el conocimiento (...)” (Stenhouse, 2004, p. 93 ), como abono que nutre la formación del colectivo estudiantil.

\section{Conclusiones}

En la actualidad, el contexto universitario público costarricense se ha de transformar en un espacio vivo, en donde se logre aprovechar el aprendizaje para una formación activa, producto de la participación conjunta para la construcción del saber con funcionalidad, atendiendo las necesidades que imponen el desarrollo personal y profesional de los individuos.

Todo cambio en la sociedad impacta, sustancialmente, los contextos de aprendizaje educativos y los procesos de socialización de los ciudadanos, ya que los asuntos que nutren y dan vida a los aprendizajes suponen derivarse de las realidades acontecidas en el entorno y, por supuesto, al estar en constante transformación, demandan un trato igualmente dinámico para su efectividad.

Entonces, entre los desafíos sustanciales de la educación pública universitaria de Costa Rica, están:

- Garantizar una transformación ética de la organización como parte sustancial del proceso permanente de autoeducación requerido por el colectivo social.

- Valorar, de forma continua, la incidencia que subyace entre la política curricular y las prácticas promovidas como elementos de impacto en la transformación de la cultura docente.

- Identificar la relevancia concedida a la construcción del conocimiento como fundamento de la práctica pedagógica desarrollada en sus contextos de aprendizaje.

- Replantearse, utilizando lo anterior a manera de insumo, los enfoques de enseñanza tradicionalmente promovidos, por unos de profunda y consensuada reflexión y transformación, en donde la dimensión ética sea la vía que conducirá al logro de una educación de calidad .

\section{Referencias bibliográficas}

Freire, P. (2002). Pedagogía de la autonomía. Buenos Aires: Siglo XXI Editores.

Gimeno, J. y Pérez, A. I. (1992). Comprender y transformar la enseñanza. Madrid: Morata.

Oliva, F. y Henson, K. T. (1989). ¿Cuáles son las competencias genéricas esenciales de la enseñanza? En J. Gimeno y A. Pérez (eds.). La enseñanza: Su teoría y su práctica (pp. 356-363). España: Ediciones Akal.

Perrenoud, P. (2004). Diez nuevas competencias para enseñar. Barcelona: Graó. 
Santos, M. A. (2001). La escuela que aprende (2 ${ }^{\mathrm{a}}$ ed.). Madrid: Morata.

Stenhouse, L. (2004). La investigación como base de la enseñanza (5 $5^{\mathrm{a}}$ ed.). Madrid: Ediciones Morata.

Tedesco, J. C. (1999). El nuevo pacto educativo (3 ${ }^{\text {ra }}$ ed). España: Anaya.

Torres, J. (2005). El curriculum oculto (8 ${ }^{\mathrm{a}}$ ed). Madrid: Morata.

Torres, J. (2006). Globalización e interdisciplinariedad: el curriculum integrado (4⿳亠丷厂 ed.). Madrid: Morata.

Zabala, A. (1999). Función social de la enseñanza y enfoque globalizador (Cap. 2). En A. Zabala (aut.). Enfoque y pensamiento complejo. Una respuesta para la comprensión e intervención en la realidad (pp. 35-66). Barcelona: Graó. 